\title{
Warum Medizin studieren?
}

\section{Beweggründe von Studierenden für ein Medizinstudium}

\author{
J. C. Becker ${ }^{1 *}$, D. Burghaus ${ }^{1 *}$, K. Kappes ${ }^{2}$, M. Heue ${ }^{3}$, A. Liebelt ${ }^{1}$, A. Kindler Röhrborn ${ }^{4}$, B. Pfleiderer ${ }^{2}$
}

\section{Zusammenfassung}

Hintergrund | Trotz stetig steigender Arztzahlen ist der Ärztemangel nach wie vor eines der größten Probleme im bundesdeutschen Gesundheitswesen. Ziel der vorliegenden Studie ist eine über den bisherigen Kenntnisstand hinausgehende, detaillierte und geschlechterspezifische Analyse aktueller Beweggründe, Medizin zu studieren, um daraus Implikationen für die Sicherstellung der ärztlichen Versorgung der kommenden Jahre abzuleiten.

Methode I Medizinstudierende an den Standorten Duisburg-Essen und Münster wurden unter Verwendung eines Online-Fragebogens nach ihren Beweggründen für die Entscheidung zum Medizinstudium gefragt. 13 Aussagen konnten mittels einer 5-stufigen Likert-Skala hinsichtlich ihrer Wichtigkeit bewertet werden. Die Auswertung erfolgte deskriptiv unter Berücksichtigung von Alter, Geschlecht, Standort und Studienabschnitt bzw. analytisch nach Dichotomisierung der Daten in Ablehnung und Zustimmung als Subgruppenanalyse mittels logistischer Regression.

Ergebnisse I An der freiwilligen Umfrage nahmen 1545 Studierende teil (64,5\% weiblich).
„Vielfältige Arbeitsbereiche“, „Abwechslungsreiche Tätigkeit“, „Patienten helfen“, „Wissenschaftliches Interesse“ und „Gute Berufsaussichten“ waren in absteigender Häufigkeit die am meisten angegebenen Beweggründe für ein Medizinstudium. Der Aspekt des Helfens war für Frauen - absolut gesehen - wichtiger als für Männer, diese bewerteten karriereorientierte Punkte wie Ansehen, Verdienst etc. höher als Frauen dies taten. Lediglich ca. 8\% der Befragten, unabhängig vom Geschlecht, sahen in einer „guten Vereinbarkeit von Familie und Beruf" einen Beweggrund für die Entscheidung zum Medizinstudium.

Schlussfolgerung I Ergebnisse dieser Erhebung können perspektivisch dazu beitragen, ärztliche Arbeitsfelder so zu gestalten, dass diese auch für die heranwachsende Medizinergeneration attraktiv bleiben: eine anspruchsvolle, erfüllende und familienkompatible Tätigkeit, mit der Option, „Karriere zu machen“ für diejenigen, die dies wünschen - unabhängig vom Geschlecht. Des Weiteren sollte man darüber hinausgehend studiums- und berufsbegleitende Maßnahmen (z.B. „Coaching on the job“) zur besseren Passung von geschlechtsspezifischen Beweggründen und Karrierepfaden anbieten.

\section{Einleitung}

Der Ärztemangel ist - trotz stetig steigender Arztzahlen - nach wie vor eines der größten Probleme im bundesdeutschen Gesundheitswesen. Eine Besserung ist nicht in Sicht. Die Ursachen des Ärztemangels sind vielfältig und er ist nicht, bzw. allenfalls marginal, durch Studienabbrecher, geringe Absolventenzahlen, Abwanderungen von Ärzten in das Ausland oder nichtkurative Tätigkeitsbereiche erklärbar [1]. Vielmehr wird neben demografischen Faktoren und dem medizinischen Fortschritt als weiterer Grund die zunehmende Anzahl von Ärztinnen, die vor allem in Teilzeit tätig sind, genannt [2]. So ist die Zahl der berufstätigen Ärztinnen in der Zeit von 19912011 um ca. 83\% gestiegen, die der Ärzte lediglich um ca. $18 \%$ [3].

Unabhängig vom Geschlecht legt die jetzige Generation, die sogenannte Generation Y, ganz unab- hängig vom gewählten Studienfach mehr Wert auf Freizeit in Kombination mit einem allgemeinen Trend zur Arbeitszeitverkürzung, einer besseren Vereinbarkeit von Familie und Beruf sowie einer gesteigerten Zufriedenheit am Arbeitsplatz [4] als dies vorherige Generationen taten. Dies zeigt aber auch, dass das Geschlecht zwar ein Faktor, aber nicht die einzige Ursache aller Probleme sein kann und dass diese Entwicklungen es mittelfristig unabdingbar machen, tradierte Strukturen aufzubrechen und sowohl das Medizinstudium, insbesondere aber auch die Organisation und Rahmenbedingungen ärztlicher Arbeit noch weiter an neue Gegebenheiten anzupassen.

Für eine ausgewogenere Diskussion ist eine Kenntnis von geschlechtsspezifischen

- Karrierezielen,

- Bedürfnissen,

- Wünschen und

- Motivationen
Institute

1 Institut für Ausbildung und Studienangelegenheiten, Westfälische

Wilhelms-Universität Münster

2 Institut für Klinische Radiologie, Universitätsklinikum Münster

3 Medizinische Fakultät, Universität DuisburgEssen, Essen

4 Institut für Pathologie, Universitätsklinikum Duisburg-Essen, Essen

* Geteilte Erstautorenschaft

\section{Korrespondenz}

Dr. med. Jan C. Becker Institut für Ausbildung und Studienangelegenheiten, Westfälische WilhelmsUniversität Münster Albert-Schweitzer-Campus 1, Gebäude A6 48149 Münster Fon: $0251 / 8358939$ Fax: $0251 / 8358933$ jan.becker@uni-muenster.de 


\begin{tabular}{|llllll|}
\hline Kenngröße & $\begin{array}{l}\text { Grundgesamtheit } \\
\text { (GG) }\end{array}$ & \multicolumn{2}{c}{ Studie gesamt } & & \multicolumn{2}{l|}{ Studie Beweggründe f. Medizinstudium } \\
\hline Studierende & 3496 & Gesamt Studie & \% der GG & Teilnehmer & \% der GG \\
\hline Davon männlich & & 1691 & 48,37 & 1545 & 44,19 \\
\hline Davon Münster & 1820 & 604 & & 584 & \\
\hline Davon Essen & 1676 & 1000 & 54,95 & 914 & 50,22 \\
\hline Davon Vorklinik & 1229 & 691 & 41,23 & 632 & 37,71 \\
\hline Davon Klinik & 1810 & 555 & 45,16 & 483 & 39,30 \\
\hline Davon PJ & 457 & 831 & 45,91 & 780 & 43,09 \\
\hline
\end{tabular}

Tab. 1 Demografische Zusammensetzung der Teilnehmer an dem Studien-Teilaspekt „Beweggründe für ein Medizinstudium" im Vergleich zu denen der gesamten Studie (hier nicht ausgewertet) bzw. der Grundgesamtheit. Angaben als absolute Werte und relative Häufigkeiten in der jeweiligen Gruppe in Prozent. des medizinischen Nachwuchses notwendig. Während erstere Aspekte [4-6] bereits detailliert untersucht wurden, liegen zu der Frage der Motivation für die Entscheidung zum ärztlichen Beruf bisher relativ wenige Daten vor. In einer Befragung von Erstsemestern aus dem Jahr 2004 konnten Sönnichsen et al. [7] herausarbeiten, dass

- „Arbeiten mit Menschen“,

- „Helfen“ und

- „Anspruchsvolle Tätigkeit“

die am häufigsten genannten Studienbeweggründe waren. Geschlechtsunterschiede zeigten sich, hinsichtlich der beiden erstgenannten Gründe (Frauen > Männer), wohingegen Männer Aspekte wie Karrieremöglichkeiten, Verdienst und gesellschaftliches Ansehen wichtiger einstuften als Frauen dies taten. Ähnliches wurde auch im Bericht zum EMS (Eignungstest für das Medizinstudium in der Schweiz und Österreich) aus dem Jahr 2010 [8] an einem österreichischen und schweizerischen Kollektiv vermerkt.

Ziel der vorliegenden, an zwei verschiedenen medizinischen Fakultäten durchgeführten, explorativen Studie ist eine über den bisherigen Kenntnisstand hinausgehende, detaillierte und geschlechterspezifische Analyse aktueller Beweggründe, Medizin zu studieren, um daraus Implikationen zur Sicherstellung der ärztlichen Versorgung der kommenden Jahre abzuleiten.

\section{Methode}

Im Rahmen der Querschnittsstudie „Geschlechtersensible Lehrmodule in der Medizin“ (www. gendermedlearning.de) wurden 3496 Studierende der medizinischen Fakultäten Duisburg-Essen und Münster als unabhängiger Teilaspekt zu ihren Beweggründen für die Aufnahme des Medizinstudiums befragt („Was waren Ihre wesentlichen Beweggründe für die Aufnahme eines Medizinstudiums?"). Ihnen wurden 13 mögliche Gründe vorgegeben (Online-Supplement, \ sAbb. 1), die sie mittels einer 5-stufigen Likert-Skala (von -2 : unzutreffend bis +2 : zutreffend) bewerten konn- ten. Die Konzeption des der Umfrage zugrundliegenden Fragebogens (s. Supplement, \sAbb. 1) erfolgte in einem interdisziplinären Team aus

- Medizinern,

- Naturwissenschaftlern,

- Psychologen und

- Soziologen.

Nach Genehmigung des Vorhabens durch die Ethikkommission der Medizinischen Fakultät Münster / Ärztekammer Westfalen-Lippe und nach einer Pilot- bzw. Testphase wurde die Umfrage online mittels des an beiden Standorten routinemäßig eingesetzten Online-Evaluationsprogramms EVAluna ${ }^{\circledR}$ in der Zeit vom 08.11. bis 29.11.2011 durchgeführt. Dazu wurden E-Mails mit einem individuellen, aber anonymisierten Link zu der webbasierten Umfrage an die o. g. Zielgruppen verschickt. Zur Steigerung der Motivation der Studierenden an der Umfrage teilzunehmen, wurden im Vorfeld Werbemaßnahmen durchgeführt und Preise ausgesetzt. Die Rücklaufquote lag im Hinblick auf diese Fragestellung bei $44,2 \%$ (gesamte Studie $48,4 \%$ ) was einer Gesamtstichprobengröße von $n=1545$ (gesamte Studie: N=1691) entspricht; eine detaillierte Aufstellung der Zusammensetzung der Teilnehmergruppen zeigt $\bullet$ Tab 1 .

Die anschließende statistische Auswertung erfolgte unter Verwendung von SPSS ${ }^{\circledR} 21$ (IBM, Ehningen, Deutschland). Ein Großteil der Analysen wurde mittels multifaktorieller Varianzanalyse unter Berücksichtigung der Haupteffekte entsprechender Einflussfaktoren (Geschlecht, Studienabschnitt, Standort) sowie deren Interaktionen durchgeführt. Unterschiede wurden bei einem $\mathrm{p}<0,05$ und $\mathrm{p}<0,01$ als signifikant, ab einem $\mathrm{p}<0,001$ als hochsignifikant angesehen.

Um einen Vergleich der aktuellen Ergebnisse mit vorhergehenden Untersuchungen zu ermöglichen, wurden die mittels Likert-Skala erhobenen Daten in einen Wertebereich von 1-5 transformiert. Zudem wurden, um Subgruppen besser identifizieren und charakterisieren zu können, die 
Antworten der Befragung dichotomisiert („Ablehnung“ $[-2,-1,0]$ und „Zustimmung“ $[+1,+2]$ ).

Mit diesen Daten wurde des Weiteren eine logistische Regression durchgeführt, um zu klären, welche Variablen einen Einfluss auf die Vorhersage der abhängigen Variablen haben. Durch Verwendung der Rückwärtsselektion wurden schrittweise Variablen aus dem Modell entfernt, die keinen oder einen nur marginalen Einfluss haben.

\section{Ergebnisse}

Grundsätzlich zählten in absteigender Reihenfolge

- „Vielfältige Arbeitsbereiche“,

- „Abwechslungsreiche Tätigkeit“,

- „Patienten helfen“,

- „Wissenschaftliches Interesse“ und

- „Gute Berufsaussichten“

zu den Beweggründen für ein Medizinstudium, denen im Mittel am deutlichsten zugestimmt wurde. Auf Zustimmung trafen ferner die Aussagen „Hohe Verantwortung“, „Gesellschaftliches Ansehen“ und „Guter Verdienst“. „Keine speziellen Gründe“, „Ärzte in der eigenen Familie“ sowie „Vereinbarkeit von Familie und Beruf“ fanden die geringste Zustimmung, gefolgt von „Als Hausarzt arbeiten“ und „Gutes Abiturergebnis“. Die Ergebnisse sind detailliert in $>$ sTab. 1 zusammengefasst.

Geschlecht, Studienabschnitt, Studienstandort | Hochsignifikante geschlechtsspezifische Unterschiede fanden sich für die Beweggründe

- „Gesellschaftliches Ansehen“(Männer > Frauen),

- „Gute Berufsaussichten“ (M>F),

- „Hohe Verantwortung“ ( $M>F)$ sowie für

- "Patienten helfen“ ( $\mathrm{F}>\mathrm{M})$.
Signifikante Unterschiede konnten für „Guter Verdienst“ (M>F) und „Abwechslungsreiche Tätigkeit“ (F>M) dokumentiert werden. Hinsichtlich der von beiden Geschlechtern insgesamt ablehnend beurteilten Punkte wurden Unterschiede für die Punkte „Keine speziellen Gründe“ $(\mathrm{M}>\mathrm{F})$ und „Gutes Abiturergebnis“ ( $\mathrm{F}>\mathrm{M})$ gefunden. Die Ergebnisse sind in $>$ Abb. 1 zusammengefasst.

Der Studienabschnitt hingegen zeigte bei folgenden Gründen einen hochsignifikanten Einfluss:

- „Patienten helfen“ (Vorklinik [VK]> Klinik

$[\mathrm{K}]>$ Praktisches Jahr [PJ]) und

- "Gute Vereinbarkeit von Familie und Beruf“ ( $\mathrm{VK}>\mathrm{K}>\mathrm{PJ}$ ).

Signifikante Differenzen ergaben sich ebenfalls hinsichtlich der Aussagen

- „Guter Verdienst“ (VK>K>PJ),

- "Wissenschaftliches Interesse“ (VK $>\mathrm{K}>\mathrm{PJ})$ und

- „Als Hausarzt arbeiten“( $\mathrm{VK}>\mathrm{K}>\mathrm{PJ})$.

Alle Aussagen fanden mit zunehmendem Studienverlauf (Vorklinik>Klinik>PJ) im Durchschnitt weniger Zustimmung. Im Gegensatz dazu wurde der Aussage „keine speziellen Gründe“ $(\mathrm{VK}<\mathrm{K}<\mathrm{PJ})$ mit zunehmendem Studienabschnitt weniger deutlich widersprochen ( $\triangleright$ Abb. 2).

Standortunterschiede betreffend war die Ablehnung der Essener Studierenden hochsignifikant stärker hinsichtlich der Aussagen

- "Gute Vereinbarung von Familie und Beruf“ (Münster > Essen) und

- „Gutes Abiturergebnis“ (Münster $>$ Essen) als Beweggründe für die Aufnahme des Medizinstudiums. Im Mittel hat das „Gesellschaftliche Ansehen“ (Münster > Essen) für Münsteraner Studierende signifikant höhere Bedeutung als für die Kommilitonen aus Essen.

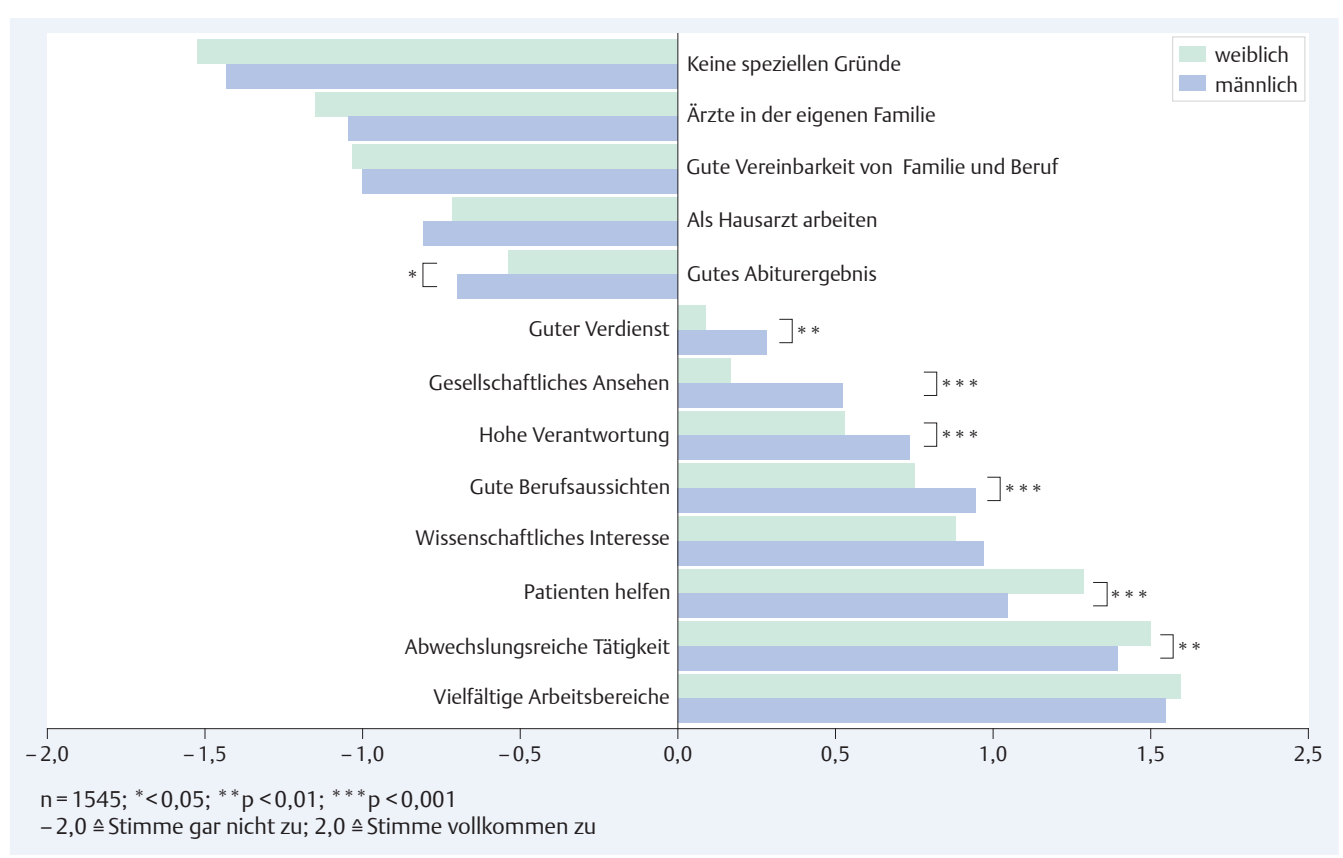

Abb. 1 Beweggründe für die Aufnahme des Medizinstudiums in Abhängigkeit vom Geschlecht. 
Abb. 2 Beweggründe für die Aufnahme des Medizinstudiums in Anhängigkeit vom Studienabschitt.

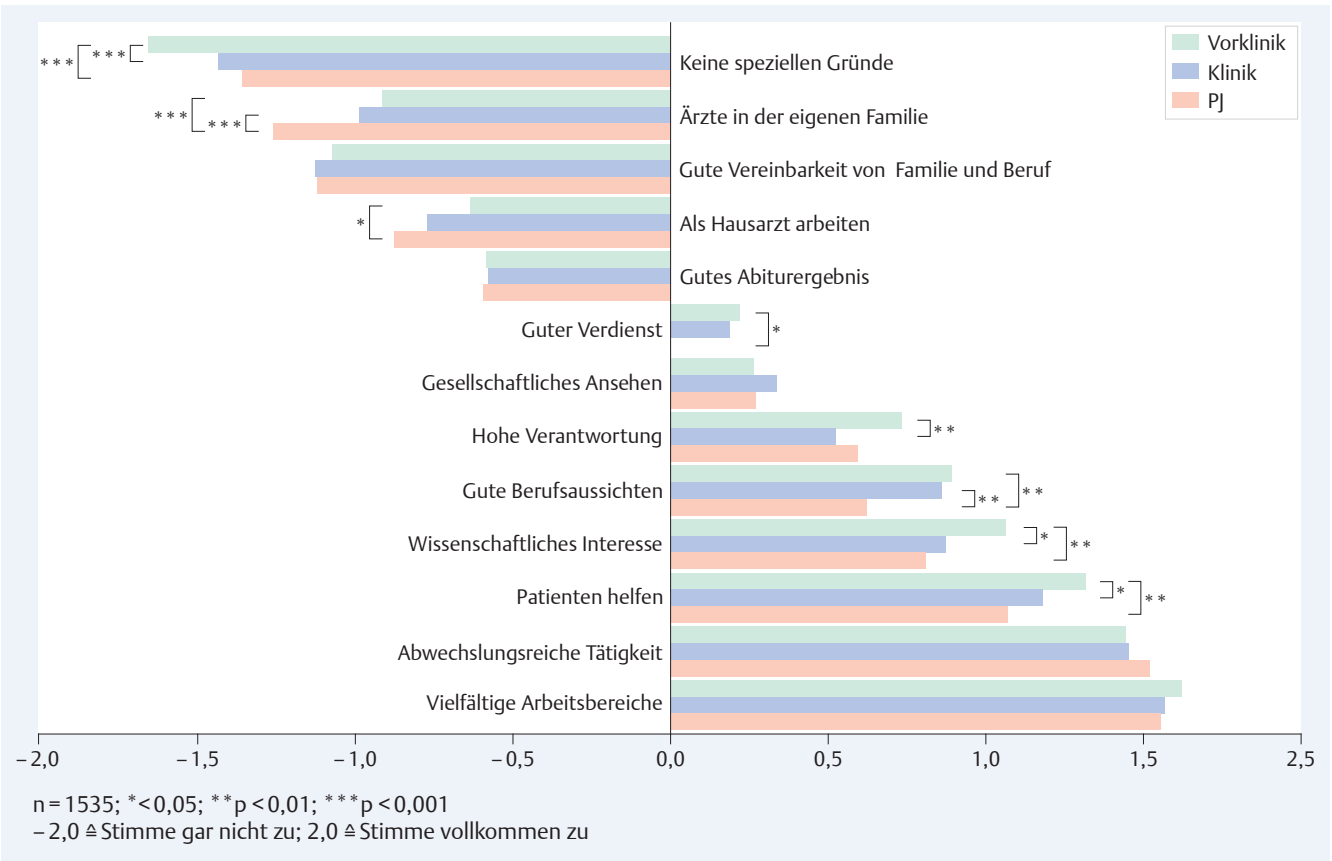

Es gab keine signifikanten Interaktionen zwischen den Einflussfaktoren Geschlecht, Studienabschnitt und Standort.

Subgruppenanalyse I 107 Studierende (6,9\%) stimmten der Aussage „Keine speziellen Gründe/ Ich wusste nicht, was ich sonst machen sollte“ $(+1$ und +2$)$ zu. Im Vergleich zu den 1433 Studierenden, die hier neutral oder ablehnend $(-2,-1,0)$ antworteten, waren für diese Gruppe grundlegende Aspekte eines Medizinstudiums bzw. des ärztlichen Berufes wie „Patienten helfen“, „Hohe Verantwortung“ und „Vielfältige Arbeitsbereiche“ oder „Wissenschaftliches Interesse“ als Beweggrund für die Aufnahme eines Medizinstudiums hochsignifikant weniger wichtig. Hingegen wurde das Motiv „Gutes (eigenes) Abiturergebnis“ deutlich höher bewertet.

Die Zustimmung zu karriereorientierten Parametern wie

- „Gute Berufsaussichten“ (insges. 71\%, M>F),

- „Hohe Verantwortung“ (62\%, M > F),

- "Gesellschaftliches Ansehen“ (49\% M>F) und

- "Guter Verdienst“ (44\% (M > F)

war generell relativ hoch - insbesondere aber unter den Männern. Bei Zustimmung zu einem der o.g. Parameter war die Chance zur Zustimmung zu den anderen beiden Punkten signifikant höher im Vergleich zu denjenigen, die jeweils neutral oder ablehnend antworteten (Ausnahme: „Hohe Verantwortung“ - hier nur im Hinblick auf „Gesellschaftliches Ansehen“). Ein Einfluss auf altruistische Beweggründe („Patienten helfen“) bei Zustimmung zu o.g. karriereorientierten Motiven lag nicht vor. Die Chance, letzteren Aspekt als Beweggrund für ein Medizinstudium zu nennen, war für Studentinnen deutlich höher.
Für 383 Studierende (25\%) war ein „Gutes (eigenes) Abiturergebnis“ ein Grund, Medizin zu studieren. In dieser Gruppe war die Chance zur Zustimmung zu den Beweggründen „Abwechslungsreiche Tätigkeit“ und „Hausärztlich arbeiten “ signifikant niedriger. Höher war sie in Bezug auf die Motive „Keine speziellen Gründe“, „Gesellschaftliches Ansehen“, „Gute Berufsaussichten“, „Gute Vereinbarkeit von Familie und Beruf“ und „Ärzte in der eigenen Familie“. Letzterer Punkt war für knapp 20\% der Befragten ein Grund für die Aufnahme eines Medizinstudiums.

Die Zustimmung zum Beweggrund „Als Hausarzt arbeiten“ lag bei $15 \%$ (225 von 1318); für entsprechende Studierende spielte das „eigene Abiturergebnis“ eine geringere, der Aspekt des „Helfens“ und eine „gute Vereinbarkeit von Familie und Beruf" hingegen eine wichtigere Rolle.

Lediglich 129 Studierende (8\%) sahen in einer „Guten Vereinbarkeit von Familie und Beruf“ einen Grund, Medizin zu studieren. In dieser Gruppe war die Chance zur Zustimmung von karriereorientierten („Gute Berufsaussichten“; „Guter Verdienst“, „Gutes Abiturergebnis“) als auch altruistischen Beweggründen („Patienten helfen“; „Als Hausarzt arbeiten“) höher. Die Ergebnisse der Subgruppenanalyse sind in $>$ Tab. 2 zusammenfasst.

\section{Diskussion}

Die hier vorgestellten, an zwei verschiedenen medizinischen Fakultäten erhobenen Daten, geben einen repräsentativen Überblick über aktuelle Beweggründe von Medizinstudierenden für die Wahl ihres Studiums. Dabei spielen neben einer 
abwechslungsreichen Tätigkeit vor allem altruistische Gründe, gute Berufsaussichten/Karrieremöglichkeiten und wissenschaftliches Interesse eine gewichtige Rolle. Wie bereits in vorherigen Studien beschrieben, gibt es hochsignifikante Geschlechtsunterschiede: Für Frauen hat der Aspekt der Fürsorge für den Patienten höhere Bedeutung als für Männer, diese gewichten wiederum karriereorientierte Parameter wie gesellschaftliches Ansehen, berufliche Perspektiven, Verdienstmöglichkeiten und Verantwortung höher als das weibliche Geschlecht. Unseres Wissens konnten diese Unterschiede auch erstmals quantifiziert werden.

Vergleicht man die Ergebnisse dieser Erhebung mit einer vorangegangenen Befragung mit Studienanfängern aus dem Jahr 2004 (Sönnichsen et al. 2004 [7]), so fällt auf, dass neben dem Grund „Wissenschaftliches Interesse“ vor allem Frauen karriereorientierten Motiven aktuell deutlicher zustimmen als zuvor. Im Gegensatz dazu bewerteten die Studierenden in der vorliegenden Umfrage die Beweggründe „Patienten helfen“ und „Vereinbarkeit von Familie und Beruf" tendenziell weniger positiv bzw. widersprachen deutlicher. Interessanterweise waren in der aktuellen Erhebung die Gründe „Vielfältige Arbeitsbereiche“ und „Abwechslungsreiche Tätigkeit“ die am wichtigsten gewerteten Motive, während in der an Studienanfängern durchgeführten Studie [7] die Aspekte „mit Menschen arbeiten“ und „anderen Menschen helfen" die höchsten Zustimmungen erfuhren (vor Gründen wie „intellektuelle“ oder „anspruchsvolle Tätigkeit“). Die hohe Bewertung der erstgenannten Punkte ist gut vereinbar mit den Einstellungen der Generation Y zur Arbeit: „Leben beim Arbeiten“, „Flexibilität und Mobilität sind wichtig“" [4]. Einschränkend muss allerdings festgestellt werden, dass in der Studie von Sönnichsen et al. [7] lediglich Studienanfänger befragt wurden, hier Studierende aller Semester.

Zur Beunruhigung Anlass geben sollte die Tatsache, dass dem Motiv „Gute Vereinbarkeit von Familie und Beruf" sowohl im Vergleich zu der o.g. Studie, insbesondere aber bei Studierenden im fortgeschrittenem Studienabschnitt deutlicher widersprochen wurde. Gerade dieser Aspekt ist gemäß einer der größten bundesweiten Studierendenbefragungen für den ärztlichen Nachwuchs von größter Bedeutung [5], entsprechend ergibt sich hier ein besonders großes Optimierungspotenzial. Unter der Einschränkung, dass es sich bei der vorliegenden Studie um eine Querschnittserhebung handelt, ist ein besonders markanter Sprung zwischen klinischem Studienabschnitt und dem PJ erkennbar, der vermuten lässt, dass hier eine Konfrontation mit der „Realität“ stattfand.

Nicht nur die Zustimmung zu dem im vorherigen Absatz diskutierten Beweggrund nahm im Vergleich der Studienabschnitte (Vorklinik > Kli- nik $>$ PJ) ab, sondern auch zentrale Aspekte wie „Patienten helfen“ und „Wissenschaftliches Interesse“ oder „Guter Verdienst“. Die im Mittel zu konstatierende Ablehnung des Motivs „hausärztlich Arbeiten“ verstärkte sich analog dazu. Wie oben erwähnt, handelt es sich bei der aktuellen Studie lediglich um eine Momentaufnahme, die naturgemäß Aussagen über Meinungsänderungen im Studienverlauf nur sehr eingeschränkt ermöglicht.

Obwohl explizit nach den Gründen für die Entscheidung zur Aufnahme eines Medizinstudiums gefragt wurde, erscheint allerdings eine Beeinflussung durch die aktuelle Situation/den Studienabschnitt als höchst wahrscheinlich gegeben. Zur weiteren Untersuchung dieser Fragestellungen sind jedoch longitudinale Studien unabdingbar. Bestätigt sich o.g. Tendenz, so könnte dies ein Ausdruck enttäuschter Erwartungen oder falscher Vorstellungen vom Medizinstudium bzw. vom ärztlichen Beruf sein.

Im Fokus der öffentlichen Kritik steht insbesondere der für ein Medizinstudium notwendige hohe Numerus clausus. In der aktuellen Studie spielte für ca. $25 \%$ der Studienteilnehmer (ohne signifikante Geschlechtsunterschiede) das eigene Abiturergebnis eine Rolle bei der Studienwahl. Für entsprechende Studierende sind „Gesellschaftliches Ansehen“, „Gute Berufsaussichten“ sowie „eine Vereinbarkeit von Familie und Beruf“" wichtiger als für Kommilitonen, die die Frage nach dem Abiturergebnis als Studienmotiv neutral oder negativ beantwortet haben. Die Chance, die Frage nach hausärztlicher Tätigkeit als Studienbeweggrund positiv zu beantworten, ist in dieser Gruppe niedriger, hingegen die, der Aussage „(ich hatte) keine speziellen Gründe“ zuzustimmen, hochsignifikant höher. Derzeit ist jedoch vollkommen unklar, ob der Studienbeweggrund „Gutes (eigenes) Abiturergebnis“ - bzw. Studienbeweggründe generell - irgendeinen Einfluss auf die „Qualität“ und Berufswahl angehender Mediziner haben.

Kritisch gesehen werden sollte aber nach Ansicht der Autoren der Anteil der o.g. Gruppe bzw. diejenigen, die der Aussage „(ich hatte...) keine speziellen Gründe/ich wusste nicht, was ich sonst machen sollte“ zustimmten. In dieser Gruppe (107 Studierende, 6,9\% der Teilnehmer) war die Chance zur Zustimmung zu fundamentalen Aspekten des ärztlichen Berufes („Patienten helfen“, „Wissenschaftliches Interesse“, „Hohe Verantwortung") teils hochsignifikant geringer im Vergleich zu denjenigen, die diese Aussage neutral oder negativ bewerteten. Zwar betrifft dies nur relativ wenige Studierende und möglicherweise deutlich weniger als in anderen Studiengängen, hat aber vor dem Hintergrund des Ärztemangels und den Kosten der ärztlichen Ausbildung dennoch gesellschaftliche und sozioökonomische Bedeutung. 
Letztlich drängt sich bei der Betrachtung der Ergebnisse dieser Studie die Frage nach den Auswahlkriterien für das Medizinstudium auf: Können bzw. sollten individuelle Motivationsgründe perspektivisch bei der Auswahl zukünftiger Medizinstudierender berücksichtigt werden - mit dem Ziel einer im Hinblick auf den Ärztemangel adäquateren und zielgerichtetere Studienplatzvergabe?

Um dieser Frage im Ansatz nachgehen zu können, ist nach Ansicht der Autoren eine stärker psychologische Betrachtungsweise der Thematik notwendig: Welche Beweggründe können als Motive im psychologischen Sinne behandelt werden? Ferner, lassen sich motivations- und persönlichkeitsbasierende Profile entwickeln, die über den Auswahlprozess hinausgehend sowohl der eigenen Berufsentscheidung, als auch der individuellen Förderung während des Studiums und/oder der Weiterentwicklung im Arztberuf dienlich sein können?

Konkret könnten diese Überlegungen mittels Kenntnissen und Methoden der Motivationspsychologie überprüft und umgesetzt werden, die sich im Wesentlichen der „Zielgerichtetheit menschlichen Verhaltens" widmet [9]. Mittels psychologisch diagnostischer Instrumente lassen sich beispielsweise drei grundlegende Motive ermitteln, die sich in Anschluss-, Leistungs-, und Machtmotiv aufgliedern [10]. Daher wäre es zunächst interessant zu prüfen, inwieweit sich die hier erfragten Beweggründe der Studierenden mit den o.g. grundlegenden Motiven aus psychologischer Betrachtungsweise überschneiden.

Zusammenfassend ergibt sich unseres Erachtens ein dringender Bedarf an weiterer Forschung, gerade auch mit operanten Messmethoden, wie sie aus der Motivationspsychologie bekannt sind. Nur so wäre zu klären, ob Motivationsgründe für ein Medizinstudium auch die für den ärztlichen Beruf sind (bis hin zu der Frage: Ist der „gute“ Studierende später auch der „gute“ Arzt?) - wohlwissend, dass im Hinblick auf die Vielfältigkeit des Arztberufes jede Antwort hierauf nur eine sehr differenzielle sein kann.

Zu den Limitationen der vorliegenden Studie kann einerseits ein Selektionsbias (zuungunsten vorklinischer und zugunsten von PJ-Studierenden), andererseits die bereits oben erwähnte fehlende longitudinale Beobachtung genannt werden. Ferner kann nicht ausgeschlossen werden, dass gewisse Antworten eine soziale Erwünschtheit widerspiegeln. Teils deutliche Unterschiede je nach Studienabschnitt sowie die Art der Befragung im Rahmen einer großangelegten Genderumfrage ohne jegliche Konsequenzen (im Unterschied zu Erhebungen im Rahmen von Auswahlverfahren) sprechen allerdings dagegen. Bei dem Vergleich der aktuellen mit vorausgegangenen Studien ist die Verwendung unterschiedlicher Termini und Skalen bei der Interpretation zu berücksichtigen. Wie in Vergleichsstudien lassen auch hier gewisse vorgegebene Beweggründe unterschiedliche Interpretationen zu, beispielsweise in Bezug auf das Motiv „Vereinbarkeit von Familie und Beruf“: eine Ablehnung könnte

- Ausdruck einer Meinung sein, dass diese im ärztlichen Beruf schlecht möglich ist, oder

- dass dieser Punkt für den Studienteilnehmer keine Bedeutung bei der Wahl des Studiums gespielt hat.

Die Autoren sind sich ebenso bewusst, dass in dieser Arbeit aktuelle Debatten zu Arbeit und Geschlecht aus den Kultur- und Sozialwissenschaften nicht integriert wurden. Das wäre sicher in diesem Kontext noch von großem Interesse und auch wichtig gewesen, um das Thema von allen Seiten zu beleuchten. Allerdings hätte eine tiefergehende Auseinandersetzung den Rahmen dieser Arbeit gesprengt.

\section{Konsequenz für Klinik und Praxis}

- Frauen und Männer haben unterschiedliche Karriereziele [8] und Beweggründe für ein Medizinstudium. Beide Faktoren sollten bei Planung und Organisation ärztlicher Bildung und Arbeit berücksichtig werden.

- „Eine gute Vereinbarkeit von Familie und Beruf“ stellt für die Mehrzahl der Befragten keinen Grund für ein Medizinstudium dar. Hier besteht Optimierungsbedarf.

- Starre Weiterbildungsordnungen sollten überdacht werden. Frauen und Männer müssen die gleichen Chancen haben, Karriere zu machen. Diejenigen, die dies explizit nicht anstreben, sondern nach einer anspruchsvollen und familienkompatiblen Tätigkeit suchen, sollten entsprechende Möglichkeiten haben (z. B. ein „kleiner“ Facharzt im „fast track“ für rein konservativ tätige Ärzte, HNO-Ärzte etc.).

- Beweggründe sollten als Vorhersagemöglichkeit für die Eignung zum ärztlichen Beruf als Studienplatzauswahlkriterium überdacht werden. Dabei ist der Unterschied zwischen „Eignung für das Studium“ und „beruflicher Eignung“ tiefer zu beleuchten und zu berücksichtigen.

- Longitudinale Studien sind notwendig, um Änderungen von Beweggründen in Studium und Beruf zu untersuchen. Eventuell werden Erwartungen enttäuscht.

\section{Danksagung}

Dieses Vorhaben wurde aus Mitteln des Bundesministeriums für Bildung und Forschung und aus dem Europäischen Sozialfonds der Europäischen Union unter dem Förderkennzeichen 01 FP 1101 gefördert. Die Autoren danken Frau Dipl. Psych. Andrea Bauland für ihre Unterstützung bei der Erstellung der Datenbank. 
Tab. 2 Mittelwerte der Bewertung der vorgegebenen Studienbeweggründe mittels 5-stufiger Likert-Skala (-2 (unzutreffend) bis +2 (zutreffend)) sowie entsprechende Standardabweichungen - dargestellt als Subgruppenanalyse von Studierenden, die das jeweilige Studienmotiv in der entsprechenden Zeile als unzutreffend bzw. neutral (-2, -1, 0 = Ablehnung) oder als zu zutreffend (1, 2 = Zustimmung) bewertet haben. Signifikante Odds ratios $(O R)$ sind angegeben $(p<0,05)$. Beispiel: Studierende, die einem „guten Abiturergebnis“ als Studienbeweggrund zugestimmt haben ( $n=383$ ), haben eine 2,86 mal höhere Chance auch dem Studienbeweggrund „Gesellschaftliches Ansehen“ zuzustimmen als diejenigen, die den Studienbeweggrund „Gutes Abiturergebnis“" neutral oder ablehnend bewerteten ( $n=1158)$.

\begin{tabular}{|c|c|c|c|c|c|c|c|c|c|c|c|c|c|c|c|}
\hline & & $=$ & 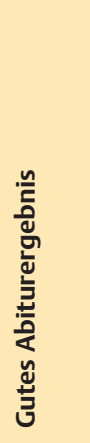 & 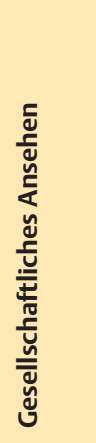 & 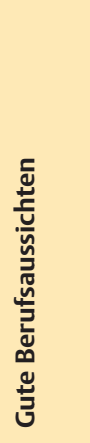 & 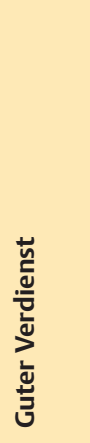 & 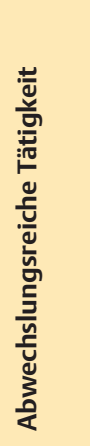 & 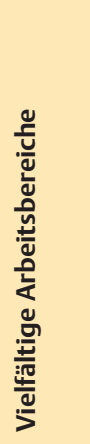 & 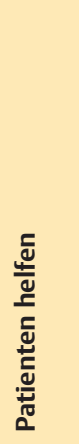 & 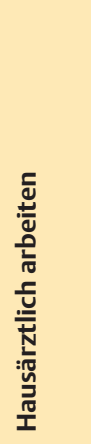 & 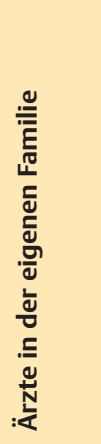 & 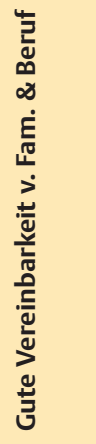 & 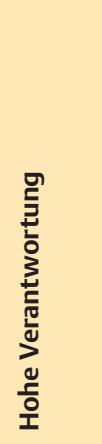 & 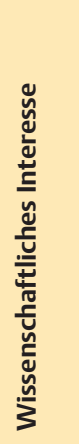 & 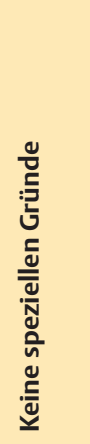 \\
\hline \multirow{6}{*}{ 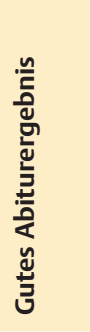 } & \multirow{2}{*}{$\begin{array}{l}\text { Zustim- } \\
\text { mung }\end{array}$} & \multirow[t]{2}{*}{383} & 1,32 & 0,81 & 1,14 & 0,51 & 1,35 & 1,58 & 1,11 & $-0,82$ & $-0,93$ & $-0,87$ & $-0,55$ & 0,93 & $-1,07$ \\
\hline & & & 0,47 & 0,95 & 0,85 & 1,04 & 0,84 & 0,73 & 0,94 & 1,09 & 1,48 & 1,10 & 1,16 & 1,10 & 1,31 \\
\hline & \multirow{2}{*}{$\begin{array}{l}\text { Ableh- } \\
\text { nung }\end{array}$} & \multirow[t]{2}{*}{1158} & $-1,22$ & 0,13 & 0,73 & 0,04 & 1,51 & 1,58 & 1,24 & $-0,73$ & $-1,17$ & $-1,07$ & 0,62 & 0,91 & $-1,63$ \\
\hline & & & 0,85 & 1,13 & 1,02 & 1,10 & 0,71 & 0,70 & 0,87 & 1,19 & 1,29 & 0,97 & 1,06 & 1,13 & 0,83 \\
\hline & OR & & & 2,86 & 1,78 & 1,29 & 0,66 & & & 0,67 & 1,43 & 1,74 & & & 5,24 \\
\hline & Sig. & & & 0,00 & 0,00 & 0,08 & 0,04 & & & 0,04 & 0,02 & 0,01 & & & 0,00 \\
\hline \multirow{6}{*}{ 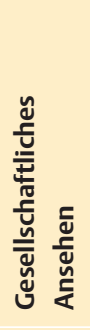 } & \multirow{2}{*}{$\begin{array}{l}\text { Zustim- } \\
\text { mung }\end{array}$} & \multirow[t]{2}{*}{756} & $-0,24$ & 1,25 & 1,15 & 0,52 & 1,44 & 1,58 & 1,22 & $-0,75$ & $-1,01$ & $-0,94$ & 0,71 & 0,91 & $-1,44$ \\
\hline & & & 1,41 & 0,44 & 0,85 & 1,04 & 0,78 & 0,70 & 0,90 & 1,16 & 1,43 & 1,05 & 1,08 & 1,11 & 1,01 \\
\hline & \multirow{2}{*}{$\begin{array}{l}\text { Ableh- } \\
\text { nung }\end{array}$} & \multirow[t]{2}{*}{787} & $-0,93$ & $-0,62$ & 0,51 & $-0,19$ & 1,49 & 1,59 & 1,20 & $-0,75$ & $-1,21$ & $-1,09$ & 0,51 & 0,93 & $-1,54$ \\
\hline & & & 1,18 & 0,76 & 1,02 & 1,06 & 0,71 & 0,72 & 0,87 & 1,18 & 1,25 & 0,96 & 1,09 & 1,14 & 0,99 \\
\hline & OR & & 2,88 & & 2,13 & 2,48 & 0,64 & & & & & & 1,66 & & \\
\hline & Sig. & & 0,00 & & 0,00 & 0,00 & 0,03 & & & & & & 0,00 & & \\
\hline \multirow{6}{*}{ 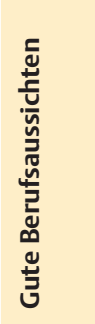 } & \multirow{2}{*}{$\begin{array}{l}\text { Zustim- } \\
\text { mung }\end{array}$} & \multirow[t]{2}{*}{1090} & $-0,44$ & 0,51 & 1,37 & 0,50 & 1,47 & 1,63 & 1,20 & $-0,76$ & $-1,09$ & $-0,89$ & 0,60 & 0,97 & $-1,46$ \\
\hline & & & 1,38 & 1,05 & 0,48 & 0,99 & 0,74 & 0,66 & 0,87 & 1,14 & 1,36 & 1,04 & 1,09 & 1,08 & 1,01 \\
\hline & \multirow{2}{*}{$\begin{array}{l}\text { Ableh- } \\
\text { nung }\end{array}$} & \multirow[t]{2}{*}{453} & $-0,95$ & $-0,23$ & $-0,46$ & $-0,66$ & 1,46 & 1,46 & 1,22 & $-0,72$ & $-1,18$ & $-1,33$ & 0,62 & 0,78 & $-1,55$ \\
\hline & & & 1,18 & 1,13 & 0,66 & 0,92 & 0,75 & 0,80 & 0,91 & 1,24 & 1,29 & 0,84 & 1,08 & 1,22 & 0,99 \\
\hline & OR & & 1,72 & 2,09 & & 12,02 & & 1,64 & & & & 2,07 & & 1,51 & \\
\hline & Sig. & & 0,00 & 0,00 & & 0,00 & & 0,05 & & & & 0,02 & & 0,01 & \\
\hline \multirow{6}{*}{ 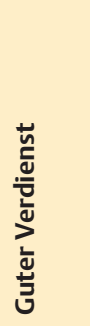 } & \multirow{2}{*}{$\begin{array}{l}\text { Zustim- } \\
\text { mung }\end{array}$} & \multirow[t]{2}{*}{675} & $-0,30$ & 0,71 & 1,36 & 1,20 & 1,42 & 1,60 & 1,21 & $-0,77$ & $-1,05$ & $-0,79$ & 0,57 & 0,91 & $-1,41$ \\
\hline & & & 1,40 & 0,99 & 0,62 & 0,40 & 0,77 & 0,67 & 0,90 & 1,14 & 1,40 & 1,07 & 1,14 & 1,10 & 1,05 \\
\hline & \multirow{2}{*}{$\begin{array}{l}\text { Ableh- } \\
\text { nung }\end{array}$} & \multirow[t]{2}{*}{868} & $-0,81$ & $-0,02$ & 0,42 & $-0,65$ & 1,50 & 1,57 & 1,20 & $-0,73$ & $-1,16$ & $-1,20$ & 0,63 & 0,92 & $-1,55$ \\
\hline & & & 1,26 & 1,12 & 1,03 & 0,74 & 0,72 & 0,74 & 0,88 & 1,19 & 1,30 & 0,91 & 1,05 & 1,15 & 0,96 \\
\hline & OR & & 1,32 & 2,44 & 11,91 & & & & & & & 2,18 & & & \\
\hline & Sig. & & 0,05 & 0,00 & 0,00 & & & & & & & 0,00 & & & \\
\hline \multirow{6}{*}{ 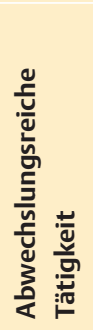 } & \multirow{2}{*}{$\begin{array}{l}\text { Zustim- } \\
\text { mung }\end{array}$} & \multirow[t]{2}{*}{1394} & $-0,63$ & 0,27 & 0,82 & 0,15 & 1,65 & 1,67 & 1,22 & $-0,74$ & $-1,12$ & $-1,04$ & 0,66 & 0,93 & $-1,53$ \\
\hline & & & 1,33 & 1,13 & 0,98 & 1,10 & 0,48 & 0,60 & 0,87 & 1,17 & 1,34 & 1,00 & 1,06 & 1,12 & 0,96 \\
\hline & \multirow{2}{*}{$\begin{array}{l}\text { Ableh- } \\
\text { nung }\end{array}$} & \multirow[t]{2}{*}{143} & $-0,20$ & 0,51 & 0,86 & 0,28 & $-0,28$ & 0,70 & 1,05 & $-0,84$ & $-1,01$ & $-0,83$ & 0,08 & 0,83 & $-1,10$ \\
\hline & & & 1,41 & 1,07 & 1,09 & 1,13 & 0,61 & 1,02 & 1,04 & 1,14 & 1,37 & 1,06 & 1,22 & 1,19 & 1,26 \\
\hline & OR & & 0,60 & 0,62 & & & & 15,25 & 1,60 & & & & 2,64 & & \\
\hline & Sig. & & 0,02 & 0,02 & & & & 0,00 & 0,05 & & & & 0,00 & & \\
\hline
\end{tabular}


Tab. 2 Fortsetzung.

\begin{tabular}{|c|c|c|c|c|c|c|c|c|c|c|c|c|c|c|c|}
\hline & & c & 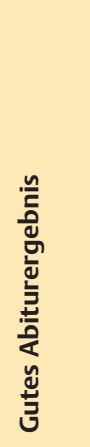 & 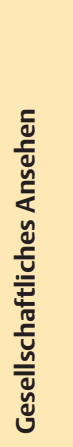 & 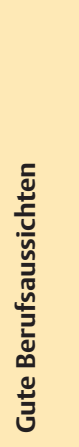 & 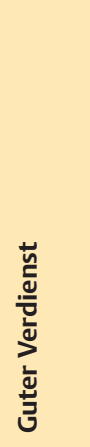 & 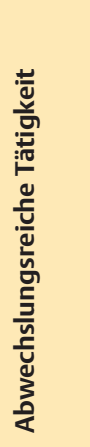 & 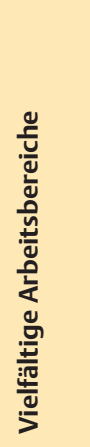 & 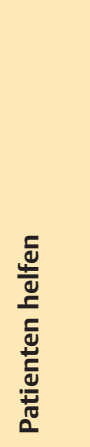 & 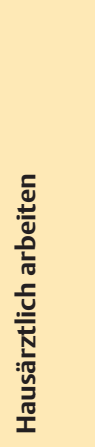 & 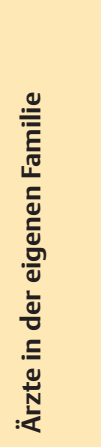 & 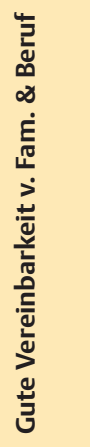 & 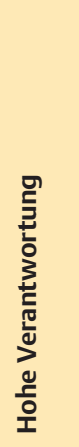 & 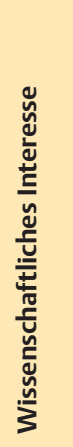 & 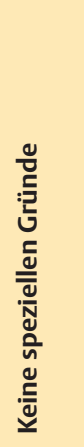 \\
\hline \multirow{6}{*}{ 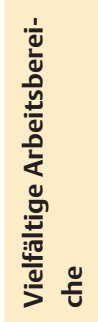 } & \multirow{2}{*}{$\begin{array}{l}\text { Zustim- } \\
\text { mung }\end{array}$} & \multirow[t]{2}{*}{1435} & $-0,60$ & 0,29 & 0,84 & 0,17 & 1,53 & 1,73 & 1,21 & $-0,75$ & $-1,12$ & $-1,03$ & 0,63 & 0,94 & $-1,51$ \\
\hline & & & 1,34 & 1,12 & 0,99 & 1,11 & 0,66 & 0,45 & 0,89 & 1,16 & 1,34 & 1,00 & 1,08 & 1,11 & 0,98 \\
\hline & \multirow{2}{*}{$\begin{array}{l}\text { Ableh- } \\
\text { nung }\end{array}$} & \multirow[t]{2}{*}{103} & $-0,50$ & 0,35 & 0,59 & 0,05 & 0,55 & $-0,42$ & 1,17 & $-0,64$ & $-1,05$ & $-0,90$ & 0,23 & 0,62 & $-1,17$ \\
\hline & & & 1,38 & 1,19 & 1,11 & 1,07 & 1,13 & 0,66 & 0,90 & 1,25 & 1,37 & 1,13 & 1,16 & 1,25 & 1,28 \\
\hline & OR & & & & 1,76 & & 15,25 & & & & & & & 1,68 & \\
\hline & Sig. & & & & 0,02 & & 0,00 & & & & & & & 0,03 & \\
\hline \multirow{6}{*}{ 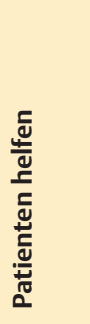 } & \multirow{2}{*}{$\begin{array}{l}\text { Zustim- } \\
\text { mung }\end{array}$} & \multirow[t]{2}{*}{1302} & $-0,61$ & 0,31 & 0,83 & 0,17 & 1,50 & 1,60 & 1,51 & $-0,67$ & $-1,11$ & $-0,98$ & 0,65 & 0,89 & $-1,56$ \\
\hline & & & 1,34 & 1,12 & 0,99 & 1,10 & 0,72 & 0,70 & 0,50 & 1,18 & 1,35 & 1,02 & 1,05 & 1,12 & 0,92 \\
\hline & \multirow{2}{*}{$\begin{array}{l}\text { Ableh- } \\
\text { nung }\end{array}$} & \multirow[t]{2}{*}{239} & $-0,47$ & 0,23 & 0,79 & 0,10 & 1,31 & 1,50 & $-0,45$ & $-1,16$ & $-1,13$ & $-1,23$ & 0,36 & 1,07 & $-1,11$ \\
\hline & & & 1,35 & 1,15 & 1,04 & 1,14 & 0,85 & 0,77 & 0,67 & 1,04 & 1,30 & 0,89 & 1,24 & 1,12 & 1,30 \\
\hline & OR & & & & & & 1,58 & & & 1,99 & & 2,23 & 1,32 & 0,74 & 0,34 \\
\hline & Sig. & & & & & & 0,04 & & & 0,01 & & 0,02 & 0,06 & 0,09 & 0,00 \\
\hline \multirow{6}{*}{ 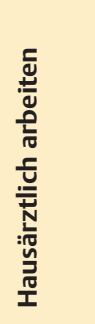 } & \multirow{2}{*}{$\begin{array}{l}\text { Zustim- } \\
\text { mung }\end{array}$} & \multirow[t]{2}{*}{225} & $-0,75$ & 0,24 & 0,68 & $-0,01$ & 1,50 & 1,52 & 1,42 & 1,27 & $-0,99$ & $-0,88$ & 0,63 & 0,75 & $-1,50$ \\
\hline & & & 1,27 & 1,17 & 1,04 & 1,13 & 0,73 & 0,77 & 0,73 & 0,45 & 1,40 & 1,07 & 1,05 & 1,15 & 0,98 \\
\hline & \multirow{2}{*}{$\begin{array}{l}\text { Ableh- } \\
\text { nung }\end{array}$} & \multirow[t]{2}{*}{1318} & $-0,56$ & 0,31 & 0,85 & 0,19 & 1,46 & 1,59 & 1,17 & $-1,09$ & $-1,13$ & $-1,04$ & 0,60 & 0,95 & $-1,49$ \\
\hline & & & 1,35 & 1,12 & 0,98 & 1,10 & 0,75 & 0,70 & 0,91 & 0,87 & 1,33 & 0,99 & 1,09 & 1,12 & 1,01 \\
\hline & OR & & 0,69 & & & 0,74 & & & 2,00 & & & 2,16 & & 0,73 & \\
\hline & Sig & & 0,05 & & & 0,06 & & & 0,01 & & & 0,00 & & 0,06 & \\
\hline \multirow{6}{*}{ 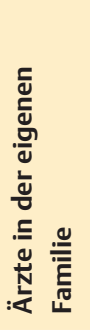 } & \multirow{2}{*}{$\begin{array}{l}\text { Zustim- } \\
\text { mung }\end{array}$} & \multirow[t]{2}{*}{303} & $-0,41$ & 0,46 & 0,92 & 0,31 & 1,47 & 1,58 & 1,23 & $-0,71$ & 1,31 & $-0,84$ & 0,64 & 0,87 & $-1,38$ \\
\hline & & & 1,42 & 1,08 & 0,98 & 1,12 & 0,73 & 0,71 & 0,88 & 1,21 & 0,46 & 1,14 & 1,10 & 1,17 & 1,07 \\
\hline & \multirow{2}{*}{$\begin{array}{l}\text { Ableh- } \\
\text { nung }\end{array}$} & \multirow[t]{2}{*}{1239} & $-0,59$ & 0,30 & 0,83 & 0,16 & 1,47 & 1,58 & 1,21 & $-0,75$ & $-1,11$ & $-1,02$ & 0,61 & 0,92 & $-1,49$ \\
\hline & & & 1,34 & 1,13 & 0,99 & 1,11 & 0,74 & 0,71 & 0,89 & 1,17 & 1,34 & 1,01 & 1,09 & 1,12 & 1,00 \\
\hline & OR & & 1,44 & 1,30 & & & & & & & & 2,17 & & & \\
\hline & Sig. & & 0,01 & 0,05 & & & & & & & & 0,00 & & & \\
\hline \multirow{6}{*}{ 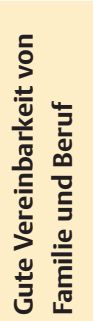 } & \multirow{2}{*}{$\begin{array}{l}\text { Zustim- } \\
\text { mung }\end{array}$} & \multirow[t]{2}{*}{129} & $-0,16$ & 0,57 & 1,33 & 0,81 & 1,42 & 1,58 & 1,44 & $-0,40$ & $-0,53$ & 1,26 & 0,60 & 0,80 & $-1,37$ \\
\hline & & & 1,43 & 1,13 & 0,82 & 0,97 & 0,80 & 0,71 & 0,85 & 1,22 & 1,67 & 0,44 & 1,11 & 1,18 & 1,11 \\
\hline & \multirow{2}{*}{$\begin{array}{l}\text { Ableh- } \\
\text { nung }\end{array}$} & \multirow[t]{2}{*}{1412} & $-0,63$ & 0,27 & 0,78 & 0,10 & 1,47 & 1,58 & 1,19 & $-0,78$ & $-1,16$ & $-1,23$ & 0,61 & 0,93 & $-1,50$ \\
\hline & & & 1,33 & 1,12 & 1,00 & 1,10 & 0,74 & 0,71 & 0,89 & 1,16 & 1,30 & 0,76 & 1,09 & 1,12 & 0,99 \\
\hline & OR & & 1,79 & & 2,01 & 2,19 & & & 2,11 & 2,21 & 2,13 & & & & \\
\hline & Sig & & 0,00 & & 0,03 & 0,00 & & & 0,03 & 0,00 & 0,00 & & & & \\
\hline
\end{tabular}


Tab. 2 Fortsetzung.

\begin{tabular}{|c|c|c|c|c|c|c|c|c|c|c|c|c|c|c|c|}
\hline & & $=$ & 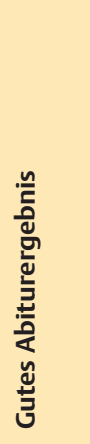 & 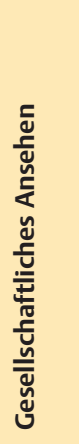 & 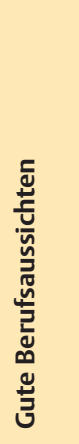 & 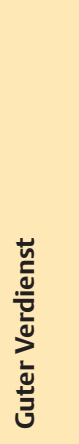 & 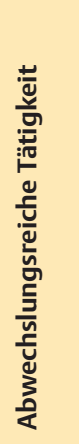 & 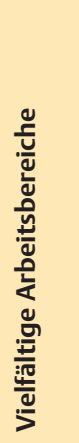 & 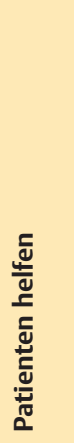 & 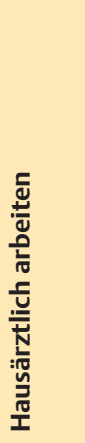 & 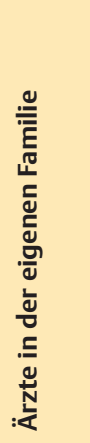 & 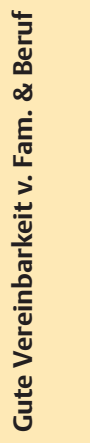 & 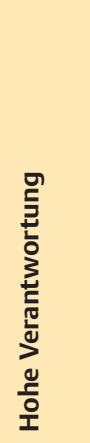 & 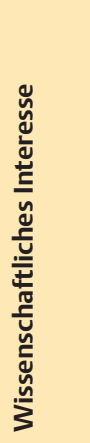 & 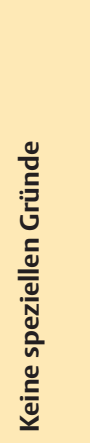 \\
\hline \multirow{6}{*}{ 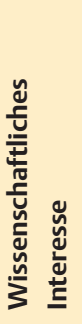 } & \multirow{2}{*}{$\begin{array}{l}\text { Zustim- } \\
\text { mung }\end{array}$} & \multirow[t]{2}{*}{1157} & $-0,56$ & 0,31 & 0,89 & 0,18 & 1,48 & 1,61 & 1,18 & $-0,78$ & $-1,13$ & $-1,04$ & 0,61 & 1,48 & $-1,54$ \\
\hline & & & 1,33 & 1,11 & 0,98 & 1,09 & 0,73 & 0,68 & 0,90 & 1,14 & 1,33 & 0,99 & 1,10 & 0,50 & 0,95 \\
\hline & \multirow{2}{*}{$\begin{array}{l}\text { Ableh- } \\
\text { nung }\end{array}$} & \multirow[t]{2}{*}{386} & $-0,69$ & 0,25 & 0,65 & 0,10 & 1,43 & 1,51 & 1,28 & $-0,65$ & $-1,06$ & $-0,95$ & 0,62 & $-0,78$ & $-1,35$ \\
\hline & & & 1,36 & 1,16 & 1,01 & 1,14 & 0,79 & 0,80 & 0,83 & 1,25 & 1,39 & 1,05 & 1,04 & 0,69 & 1,13 \\
\hline & OR & & & & 1,37 & & & 1,83 & 0,73 & 0,75 & & & & & 0,56 \\
\hline & Sig. & & & & 0,02 & & & 0,01 & 0,07 & 0,07 & & & & & 0,01 \\
\hline \multirow{6}{*}{ 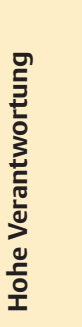 } & \multirow{2}{*}{$\begin{array}{l}\text { Zustim- } \\
\text { mung }\end{array}$} & \multirow[t]{2}{*}{960} & $-0,66$ & 0,39 & 0,82 & 0,13 & 1,62 & 1,67 & 1,29 & $-0,74$ & $-1,10$ & $-1,04$ & 1,32 & 0,92 & $-1,62$ \\
\hline & & & 1,33 & 1,10 & 0,98 & 1,12 & 0,62 & 0,63 & 0,86 & 1,19 & 1,38 & 0,98 & 0,47 & 1,12 & 0,86 \\
\hline & \multirow{2}{*}{$\begin{array}{l}\text { Able- } \\
\text { hung }\end{array}$} & \multirow[t]{2}{*}{582} & $-0,48$ & 0,14 & 0,84 & 0,21 & 1,22 & 1,44 & 1,08 & $-0,75$ & $-1,13$ & $-0,99$ & $-0,57$ & 0,91 & $-1,27$ \\
\hline & & & 1,36 & 1,15 & 1,01 & 1,09 & 0,86 & 0,80 & 0,92 & 1,14 & 1,29 & 1,05 & 0,74 & 1,14 & 1,16 \\
\hline & OR & & & 1,55 & & & 2,89 & & & & & & & & 0,45 \\
\hline & Sig. & & & 0,00 & & & 0,00 & & & & & & & & 0,00 \\
\hline \multirow{6}{*}{ 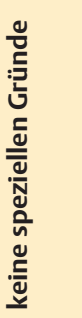 } & \multirow{2}{*}{$\begin{array}{l}\text { Zustim- } \\
\text { mung }\end{array}$} & \multirow[t]{2}{*}{107} & 0,42 & 0,34 & 0,86 & 0,21 & 1,17 & 1,36 & 0,64 & $-0,93$ & $-0,97$ & $-1,16$ & $-0,07$ & 0,58 & 1,35 \\
\hline & & & 1,43 & 1,26 & 1,10 & 1,23 & 0,94 & 0,89 & 1,25 & 1,12 & 1,42 & 1,13 & 1,30 & 1,29 & 0,48 \\
\hline & \multirow{2}{*}{$\begin{array}{l}\text { Ableh- } \\
\text { nung }\end{array}$} & \multirow[t]{2}{*}{1433} & $-0,59$ & 0,30 & 0,83 & 0,16 & 1,47 & 1,58 & 1,21 & $-0,74$ & $-1,11$ & $-1,02$ & 0,60 & 0,92 & $-1,49$ \\
\hline & & & 1,34 & 1,13 & 0,99 & 1,11 & 0,75 & 0,71 & 0,89 & 1,17 & 1,35 & 1,01 & 1,09 & 1,13 & 1,00 \\
\hline & OR & & 4,82 & & & & & 0,49 & 0,33 & & & & 0,45 & 0,52 & \\
\hline & Sig. & & 0,00 & & & & & 0,03 & 0,00 & & & & 0,00 & 0,04 & \\
\hline
\end{tabular}




\section{Literatur}

1 Schwarzer A, Gregor F. Medizinreport 2012 - Berufsstart und Berufsverlauf von Humanmedizinerinnen und Humanmedizinern. Deutsches Zentrum für Hochschul- und Wissenschaftsförderung.

http://www.dzhw.eu/pdf/22/medizinerreport 2012.pdf Letzter Zugriff am 16.09.15

2 Bundesärztekammer. Ärztemangel trotz steigender Arztzahlen - ein Widerspruch, der keiner ist 2009.

http://www.aerzteblatt.de/download/ files/2009/04/down136282.pdf Letzter Zugriff 16.09.15

3 Das Informationssystem der Gesundheitsberichterstattung des Bundes. Statistisches Bundesamt. http://www.gbe-bund.de Letzter Zugriff am 25.9.15

4 Schmidt K, Meyer ], Liebeneiner ] et al. Generation $Y$ in der HNO. Führung einer neuen Generation von Ärzten. HNO 2012; 60: 993-1002

5 Jacob R, Heinz A, Décieux JP. Berufsmonitoring Medizinstudenten. Bundesweite Befragung von Medizinstudenten, Frühjahr 2010.

http://www.kbv.de/media/sp/Studentenbefragung_ Auszaehlung_Fragebogen_bundesweit.pdf
6 Gedrose B, Wonneberger C, Jünger J et al. Haben Frauen am Ende des Medizinstudiums andere Vorstellungen über Berufstätigkeit und Arbeitsplatz als männliche Kollegen? Ergebnisse einer multizentrischen postalischen Befragung. Dtsch Med Wochenschr 2012; 137: 1242-1247

7 Sönnichsen AC, Donner-Banzhoff A, Baum E. Motive, Berufsziele und Hoffnungen von Studienanfängern im Fach Medizin. Z Allg Med 2005; 81: 222-225

8 Guntern S, Meissner E, Hänsgen KD. Studienrelevante Persönlichkeitsmerkmale für das Medizinstudium - eine Pilotstudie. Bericht zum EMS Innsbruck und Wien 2010 http://www.unifr.ch/ztd/self-assessment/ Bericht\%20SA\%202010.pdf Letzter Zugriff am 16.09.15

9 Kuhl J. Motivation und Persönlichkeit. Göttingen: Hogrefe; 2001

10 Heckhausen J, Heckhausen $\mathrm{H}$. Motivation und Handeln. Heidelberg: Springer; 2006
Interessenkonflikt

Die Autoren geben an, dass kein Interessenkonflikt besteht.

DOI 10.1055/s-0041-106581 Dtsch Med Wochenschr 2015; 140: e207-e216

(c) Georg Thieme Verlag KG . Stuttgart · New York .

ISSN 0012-0472 